\title{
Forward stagewise naïve Bayes
}

\author{
Diego Vidaurre $\cdot$ Concha Bielza $\cdot$ Pedro Larrañaga
}

Received: 10 May 2011 / Accepted: 25 October 2011 / Published online: 13 January 2012

(C) Springer-Verlag 2011

\begin{abstract}
The naïve Bayes approach is a simple but often satisfactory method for supervised classification. In this paper, we focus on the naïve Bayes model and propose the application of regularization techniques to learn a naïve Bayes classifier. The main contribution of the paper is a stagewise version of the selective naïve Bayes, which can be considered a regularized version of the naïve Bayes model. We call it forward stagewise naïve Bayes. For comparison's sake, we also introduce an explicitly regularized formulation of the naïve Bayes model, where conditional independence (absence of arcs) is promoted via an $L_{1} / L_{2}$-group penalty on the parameters that define the conditional probability distributions. Although already published in the literature, this idea has only been applied for continuous predictors. We extend this formulation to discrete predictors and propose a modification that yields an adaptive penalization. We show that, whereas the $L_{1} / L_{2}$ group penalty formulation only discards irrelevant predictors, the forward stagewise naïve Bayes can discard both irrelevant and redundant predictors, which are known to be harmful for the naïve Bayes classifier. Both approaches, however, usually improve the classical naïve Bayes model's accuracy.
\end{abstract}

Keywords Naïve bayes - Forward stagewise naïve bayes . Regularized naïve bayes $\cdot$ Redundant predictors

\footnotetext{
D. Vidaurre · C. Bielza · P. Larrañaga $(\varangle)$

Computational Intelligence Group,

Departamento de Inteligencia Artificial,

Universidad Politécnica de Madrid, Madrid, Spain

e-mail: pedro.larranaga@fi.upm.es

D. Vidaurre

e-mail: diego.vidaurre@fi.upm.es

C. Bielza

e-mail: mcbielza@fi.upm.es
}

\section{Introduction}

Bayesian network classifiers [7] are a popular supervised classification paradigm. A well-known Bayesian network classifier is the naïve Bayes [14], a simple Bayesian network classifier that assumes that the predictors or variables are independent given each class value. Despite its simplicity and strong assumptions, the naïve Bayes classifier has been proven to work satisfactorily in many domains $[4,11]$. Typically, the parameters of the naïve Bayes model are found by maximizing the joint likelihood of the model.

The naïve Bayes model's accuracy, however, declines in the presence of noisy predictors. A noisy predictor can be a predictor that either carries no useful information for the classification (irrelevant) or is strongly dependent on another predictor (redundant). Redundancy is particularly harmful, because the predictor information has double the influence than it should.

For variable selection purposes, it is common to use filtering approaches, which perform variable selection disregarding the classifier, or (greedy) wrapper algorithms, which simultaneously introduce variables into the model and iteratively estimate the parameters. We focus on the wrapper paradigm. The (stepwise) selective naïve Bayes [13] is a popular example of greedy wrapper algorithm.

Regularization techniques introduce additional information, usually to solve an ill-posed problem or to avoid overfitting. Also, by imposing certain restrictions, regularization trades off a little bias against a larger reduction in variance. $L_{1}$-regularization [15], which imposes an $L_{1}$-penalty on the parameters, is also useful for variable selection, because it drives some parameters to exactly zero.

An example of regularization within the naïve Bayes model is the $L_{1} / L_{2}$-regularized naïve Bayes, taken by van Gerven and Heskes [9], which applies optimization 
techniques to minimize the negative log-likelihood function of the data given the model plus an $L_{1} / L_{2}$-group penalty on the model complexity. This penalty encourages some predictors to be discarded. While they apply this idea only to the continuous predictor case, we extend it to deal with discrete predictors. Also, we introduce an adaptive penalty [19] that further improves the method's performance.

The main contribution of this paper, however, is a stagewise version of the selective naïve Bayes that is particularly useful when there are predictors that are relevant but, to some extent, redundant. At each iteration, instead of adding an "entire" predictor to the model, the parameters of the selected predictor are updated just a little. This method is inspired by the forward stagewise selection method for linear regression [17], which is also related to boosting and can be considered a form of regularization. We call this method forward stagewise naïve Bayes.

The remainder of the paper is organized as follows. Section 2 defines the notation and presents the basic naïve Bayes approach. Section 3 introduces some methods related to naïve Bayes, including selective naïve Bayes and the $L_{1} / L_{2}$-regularized naïve Bayes. Section 4 describes the effect of noisy (irrelevant or redundant) predictors. Section 5 introduces the forward stagewise naïve Bayes method. Section 6 discusses model selection. Section 7 outlines the set of experiments used to test the algorithms. Finally, Sect. 8 presents the conclusions and future work.

\section{Notation and classical naïve Bayes}

Let $\left\{X_{1}, \ldots, X_{p}\right\}$ be the set of $p$ predictors and $Y$ the class variable. Let $\boldsymbol{D}=\left\{\left(x_{r 1}, \ldots, x_{r p}, y_{r}\right), r=1, \ldots, N\right\}$ be the labeled data set containing $N$ instances. $\boldsymbol{X}$ denotes the $N \times p$ predictor data matrix, with elements $x_{r i}, r \in\{1, \ldots, N\}, i \in$ $\{1, \ldots, p\}$, and $\boldsymbol{y}=\left(y_{1}, \ldots, y_{N}\right)^{\mathrm{T}}$ denotes the vector of responses. We assume that the class variable, $Y$, may take values $j \in\{1, \ldots, J\}$. The objective is to learn a classifier from $\boldsymbol{D}$ so as to predict the class value for incoming data points just given by predictor values.

We assume that predictors are either discrete or continuous, although generalizations for combining the two are extremely straightforward.

When the inputs are discrete, we assume that each predictor $X_{i}$ has $M_{i}$ possible states. Assuming that the predictors are conditionally independent given the class variable, we denote their conditional probability table (CPT) as an $M_{i} \times J$ matrix $\boldsymbol{\Theta}_{i}$. Each element $\theta_{i k j}$ of $\boldsymbol{\Theta}_{i}, j \in$ $\{1, \ldots, J\}, k \in\left\{1, \ldots, M_{i}\right\}$, is the probability of the predictor $X_{i}$ taking its $k$ th state given the $j$ th class variable state, i.e., $\theta_{i k j}=P\left(X_{i}=k \mid Y=j ; \boldsymbol{\Theta}_{i}\right)$.

We assume that, when the inputs are continuous, predictors follow a Gaussian distribution within each class value.
We denote as $\boldsymbol{\mu}_{i}$ and $\sigma_{i}$ the vectors whose elements are, for each state of $Y$, the expectation and standard deviation of $X_{i}$, respectively, i.e., $X_{i} \mid Y=j \sim \mathcal{N}\left(\mu_{i j}, \sigma_{i j}\right), j \in\{1, \ldots, J\}$. We denote the conditional density function for predictor $X_{i}$, given that $Y=j$, as $f\left(x_{i} \mid j ; \mu_{i j}, \sigma_{i j}\right)$.

Let $\boldsymbol{\Theta}=\left\{\boldsymbol{\Theta}_{1}, \ldots, \boldsymbol{\Theta}_{p}\right\}, \boldsymbol{\mu}=\left\{\boldsymbol{\mu}_{1}, \ldots, \boldsymbol{\mu}_{p}\right\}$ and $\boldsymbol{\sigma}=$ $\left\{\boldsymbol{\sigma}_{1}, \ldots, \boldsymbol{\sigma}_{p}\right\}$. Likewise, we denote the whole set of predictor parameters as $\boldsymbol{\Omega}=\left\{\boldsymbol{\Omega}_{1}, \ldots, \boldsymbol{\Omega}_{p}\right\}$, where $\boldsymbol{\Omega}_{i}$ generically denotes either $\boldsymbol{\Theta}_{i}$ or $\left\{\boldsymbol{\mu}_{i}, \boldsymbol{\sigma}_{i}\right\}$. Also, we denote class prior probabilities as $\pi=\left(\pi_{1}, \ldots, \pi_{J}\right)$. Considering the predictors to be conditionally independent given the class, the full likelihood function for the naïve Bayes (NB) [14] model is defined as

$L(\boldsymbol{D} ; \boldsymbol{\Omega})=\prod_{r=1}^{N}\left[\pi_{y_{r}} \prod_{i=1}^{p} \psi\left(X_{i}=x_{r i} \mid Y=y_{r}, \boldsymbol{\Omega}_{i}\right)\right]$,

where function $\psi(\cdot)$ computes the contribution of each predictor to the full likelihood. The likelihood is thus decomposable and can be computed separately for each predictor. We now define the contribution of each predictor to the full likelihood.

Let $\boldsymbol{W}(i)$ be an $N \times M_{i}$ indicator matrix for discrete predictor $X_{i}$. For the $r$ th instance, the elements of the indicator matrix are defined as $w(i)_{r k}=1$ if $x_{r i}=k$ and $w(i)_{r k}=0$ if $x_{r i} \neq k$. Similarly, $S$ is defined as the $N \times J$ indicator matrix for class variable $Y$. Hence, the contribution of a discrete predictor $X_{i}$ and instance $r$ to the full likelihood is

$$
\begin{aligned}
\psi\left(X_{i}=x_{r i} \mid Y=y_{r}, \boldsymbol{\Omega}_{i}\right) & =P\left(X_{i}=x_{r i} \mid Y=y_{r}, \boldsymbol{\Theta}_{i}\right) \\
& =\boldsymbol{w}(i)_{r} \cdot \boldsymbol{\Theta}_{i} \boldsymbol{s}_{r}^{\mathrm{T}},
\end{aligned}
$$

where $\boldsymbol{w}(i)_{r}$. is the $r$ th row vector of $\boldsymbol{W}(i)$ and $\boldsymbol{s}_{r}$. is the $r$ th row vector of $\boldsymbol{S}$. Hence, $\boldsymbol{w}(i)_{r}$. and $\boldsymbol{s}_{r}$. are selecting the appropriate conditional probability for the $r$ th instance from $\boldsymbol{\Theta}_{i}$.

On the other hand, the contribution of a continuous predictor $X_{i}$ and instance $r$ to the full likelihood is defined as

$$
\begin{aligned}
& \psi\left(X_{i}=x_{r i} \mid Y=y_{r}, \boldsymbol{\Omega}_{i}\right)=f\left(x_{r i} \mid y_{r} ; \mu_{i y_{r}}, \sigma_{i y_{r}}\right) \\
& =\frac{1}{\sqrt{2 \pi} \sigma_{i y_{r}}} \exp -\frac{\left(x_{r i}-\mu_{i y_{r}}\right)^{2}}{2 \sigma_{i y_{r}}^{2}} .
\end{aligned}
$$

Let $\boldsymbol{\Omega}_{i}^{(0)}$ be the parameters of predictor $X_{i}$ such that they are exactly equal for all class values, that is, either $\theta_{i k j}^{(0)}$ in the discrete case or $\left\{\mu_{i j}^{(0)}, \sigma_{i j}^{(0)}\right\}$ in the continuous case are equal for all $j \in\{1, \ldots, J\}$. This is equivalent to removing predictor $X_{i}$ from the model.

To estimate a NB model, we compute the maximum likelihood estimation (MLE) of the parameters, denoted as $\hat{\boldsymbol{\Theta}}_{i}^{(1)}, \hat{\boldsymbol{\mu}}_{i}^{(1)}, \hat{\boldsymbol{\sigma}}_{i}^{(1)}$ and $\hat{\boldsymbol{\pi}}$, as 
$\hat{\theta}_{i k j}^{(1)}=\frac{N_{i j k}}{N_{j}}$,

$\hat{\mu}_{i j}^{(1)}=\frac{\sum_{r ; y_{r}=j} x_{r i}}{N_{j}}$,

$\hat{\sigma}_{i j}^{(1)}=\sqrt{\frac{\sum_{r ; y_{r}=j}\left(x_{r i}-\hat{\mu}_{i j}\right)^{2}}{N_{j}}}$,

$$
\hat{\pi}_{j}=\frac{N_{j}}{N},
$$

where $N_{i j k}$ is the number of instances in the training data set, where predictor $X_{i}$ takes the value $k$ and $Y$ takes the value $j$, and $N_{j}$ is the number of instances where $Y$ takes the value $j$.

The NB formulation for the probability of the class given the (continuous or discrete) predictors is

$$
\begin{aligned}
P(Y= & \left.j \mid X_{1}=k_{1}, \ldots, X_{p}=k_{p}, \hat{\mathbf{\Omega}}^{(1)}, \hat{\boldsymbol{\pi}}\right) \\
& \propto \hat{\pi}_{j} \prod_{i=1}^{p} \psi\left(X_{i}=k_{i} \mid Y=j, \hat{\boldsymbol{\Omega}}_{i}^{(1)}\right)=\phi_{j} .
\end{aligned}
$$

Thus, given vector $\boldsymbol{\phi}=\left(\phi_{1}, \ldots, \phi_{J}\right)$, whose components are computed with Eq. (4), the actual classification is performed by

$\hat{j}=\operatorname{maxpos}(\boldsymbol{\phi})$,

where, $\operatorname{maxpos}(\cdot)$ returns the position of the maximum element of the vector argument. Ties can be broken at random. Note that, although $\boldsymbol{\phi}$ depends on the input data configuration, it is omitted from the notation for simplicity sake.

\section{Methods related to naïve Bayes}

In this section, we introduce some existing methods related to NB: the selective naïve Bayes [13], the weighted naïve Bayes [6] and the $L_{1} / L_{2}$-regularized naïve Bayes [9]. Also, we generalize the $L_{1} / L_{2}$-regularized naïve Bayes to handle both discrete and continuous predictors and propose a simple improvement on this method.

\subsection{Existing methods}

The selective naïve Bayes (SNB) model [13] is a popular greedy, wrapper, stepwise algorithm for obtaining a NB model and performing variable selection. The SNB approach obeys Eq. (4) and, hence, makes use of the MLE. However, it is applied over only a subset of predictors. A forward greedy search finds this subset of predictors, where predictors are included in the model as long as the prediction accuracy (over training data) keeps increasing. Langley and Sage [13] also introduce a backwards search strategy, but they conclude that forward search is often more advantageous. On this ground, we use forward search in this paper.
The weighted naïve Bayes (WNB) model [6] includes all the predictors, which it weights according to their relevance for the classification. It is conceived only for discrete predictors. Weights are computed as

$w_{i}=\sqrt{\sum_{j=1}^{J} \sum_{k=1}^{M_{i}}\left[P\left(Y=j \mid X_{i}=k\right)-P(Y=j)\right]^{2}}$,

so that the resulting model is

$$
\begin{aligned}
& P\left(Y=j \mid X_{1}=k_{1}, \ldots, X_{p}=k_{p}, \boldsymbol{\Omega}\right) \propto \hat{\pi}_{j} \prod_{i=1}^{p} \\
& \quad \times \psi\left(X_{i}=k_{i} \mid Y=j, \boldsymbol{\Omega}_{i}\right)^{w_{i}}=\phi_{j} .
\end{aligned}
$$

The classification rule is the same as for NB (Eq. (5)).

Using regularization techniques, the $L_{1} / L_{2}$-regularized naïve Bayes approach $\left(L_{1} / L_{2}-\mathrm{NB}\right)$ [9], designed for continuous predictors, is formulated as the optimization problem

$$
\begin{aligned}
& \operatorname{argmin}_{\boldsymbol{\mu}, \boldsymbol{\sigma}}-\log L(\boldsymbol{D} ; \boldsymbol{\mu}, \boldsymbol{\sigma}) \\
& +\lambda \sum_{i=1}^{p} \sqrt{\sum_{j=1}^{J}\left(\mu_{i j}-\hat{\mu}_{i j}^{(0)}\right)^{2}+\sum_{j=1}^{J}\left(\sigma_{i j}-\hat{\sigma}_{i j}^{(0)}\right)^{2}}, \\
& \text { s.t. }-\sigma_{i j}<0 \quad \forall i, j,
\end{aligned}
$$

where $L(\boldsymbol{D} ; \boldsymbol{\mu}, \boldsymbol{\sigma})$ is defined in Eqs. (1) and (3) and $\lambda$ is some regularization parameter. This optimization problem has $J p$ inequality constraints.

This way, the set of parameters of each single predictor (inside the square root) forms a group. This penalty is hence a group lasso-type penalty or $L_{1} / L_{2}$-penalty [18], which is able to discard entire groups. Therefore, all the parameters $\left\{\mu_{i j}, \sigma_{i j}\right\}$ of some predictors will be prompted to be equal to $\left\{\hat{\mu}_{i j}^{(0)}, \hat{\sigma}_{i j}^{(0)}\right\}$, so that such predictors will be effectively excluded.

Note that this optimization problem is convex. First, it is well known that the Gaussian likelihood defined in Eq. (3) is log-concave and hence the negative log-likelihood is convex [3]. This can be easily proved by taking the Hessian, which is positive semidefinite and thus proves convexity. Second, the $L_{1} / L_{2}$-penalty defined in (8) is just a sum of $L_{2}$-penalties. Since the $L_{2}$-norm function is convex, it is the sum of $L_{2}$-norms. The sum of two convex functions is convex. Finally, the inequality constraint functions are just nonnegativity constraints. Therefore, problem (8) is convex and is, in fact, denoted in the standard form. Although the entire objective function is non-smooth (non-differentiable), it is composed of a smooth loss function and a block-separable penalty and, hence, the problem can be solved by unconstrained (block) coordinate gradient descent optimization [16]. The constraint can be subsumed into the penalty term by setting it to $\infty$ when $\sigma_{i j}<0$ for any pair $(i, j)$. 


\subsection{Generalized $L_{1} / L_{2}$-regularized naïve Bayes}

Now, we extend the $L_{1} / L_{2}$-NB formulation to deal with the discrete predictor case and propose an adaptive formulation of the problem for achieving better predictions.

We formulate the optimization problem for discrete predictors as

$\operatorname{argmin}_{\boldsymbol{\Theta}}-\log L(\boldsymbol{D} ; \boldsymbol{\Theta})+\lambda \sum_{i=1}^{p} \sqrt{\sum_{j=1}^{J} \sum_{k=1}^{M_{i}}\left(\theta_{i k j}-\hat{\theta}_{i j k}^{(0)}\right)^{2},}$

s.t. $\mathbf{1}_{i}^{t} \boldsymbol{\theta}_{i j}-1=0 \quad \forall i, j$,

$-\theta_{i k j}<0 \quad \forall i, j, k$,

$\theta_{i k j}-1<0 \quad \forall i, j, k$,

where, the loss function $L(\boldsymbol{D} ; \boldsymbol{\Theta})$ is defined in Eqs. (1) and (2), $\boldsymbol{\theta}_{i j}$ is the $j$ th column of $\boldsymbol{\Theta}_{i}$ and $\mathbf{1}_{i}$ is a column vector with $M_{i}$ ones. Therefore, there are $J_{p}$ equality and $\sum_{i=1}^{p} J M_{i}$ inequality constraints (each pair of inequality constraints can be subsumed in one open box constraint $\left.\theta_{i k j} \in(0,1)\right)$.

This problem is also convex and is denoted in the standard form. Since the expression in Eq. (2) is linear on $\boldsymbol{\Theta}$, it is clear that the negative log-likelihood is convex and differentiable. The penalty in the loss function is also convex (but nondifferentiable), and thus the entire loss function is convex. Both the equality and inequality constraint functions are affine. Even if we mixed both continuous and discrete predictors, the problem would still be convex. However, with the equality constraints, we cannot follow the gradient descent direction, so that (block) coordinate gradient descent optimization is not directly applicable. Instead, we take a simple approximation: starting with initial values $\Theta_{i}^{(0)}, i=1, \ldots, p$, we update $\Theta_{i}$ toward $\Theta_{i}^{(1)}$ at each iteration, while the others predictors are held fixed, until the objective function in Eq. 9 reaches a minimum. This is a just a line-search.

A possible improvement on this approach is to use an adaptive penalty, which will hopefully improve the accuracy of the estimator. In $L_{1}$-penalized linear regression [15], for example, such penalties reduce the bias and lead to a consistent estimation [19]. The innovation is to penalize each predictor variable according to its importance. Each variable penalty is thus scaled by $1 /\left|\beta_{i}^{(1)}\right|$, where $\beta_{i}^{(1)}$ is (in the $N>p$ case) the ordinary least squares regression coefficient or MLE. Note that $\left|\beta_{i}^{(1)}\right|$ is just the absolute upper bound of this coefficient in the regularized problem, i.e., the upper bound of the penalty for this variable.

We can apply an analogous idea to the $L_{1} / L_{2}$-NB formulation by computing weights $\boldsymbol{w}=\left(w_{1}, \ldots, w_{p}\right)$, for the discrete and continuous predictor cases, respectively, as

$w_{i}=\sqrt{\sum_{j=1}^{J} \sum_{k=1}^{M_{i}}\left(\hat{\theta}_{i j k}^{(1)}-\hat{\theta}_{i j k}^{(0)}\right)^{2}}$
$w_{i}=\sqrt{\sum_{j=1}^{J}\left({\hat{\mu_{i j}}}^{(1)}-{\hat{\mu_{i j}}}^{(0)}\right)^{2}+\sum_{j=1}^{J}\left({\hat{\sigma_{i j}}}^{(1)}-{\hat{\sigma_{i j}}}^{(0)}\right)^{2}}$,

so that loss functions in (9) and (8) become, respectively,

$-\log L(\boldsymbol{D} ; \boldsymbol{\mu}, \boldsymbol{\sigma})+\lambda \sum_{i=1}^{p} w_{i} \times \sqrt{\sum_{j=1}^{J} \sum_{k=1}^{M_{i}}\left(\theta_{i k j}-\hat{\theta}_{i j k}^{(0)}\right)^{2}}$,

$-\log L(\boldsymbol{D} ; \boldsymbol{\Theta})$

$+\lambda \sum_{i=1}^{p} w_{i} \sqrt{\sum_{j=1}^{J}\left(\mu_{i j}-\hat{\mu}_{i j}^{(0)}\right)^{2}+\sum_{j=1}^{J}\left(\sigma_{i j}-\hat{\sigma}_{i j}^{(0)}\right)^{2}}$.

Note that each $w_{i}$ is a tight upper bound of the penalty for predictor $X_{i}$, and here we have the parallelism with the adaptive penalty for linear regression. We call this approach adaptive $L_{1}$-regularized naïve Bayes $\left(\mathrm{a} L_{1} / L_{2}-\mathrm{NB}\right)$.

\section{Noisy predictors}

In this section, we define irrelevance and redundancy and remark on some ideas that motivate the approach introduced in Sect. 5.

We show that it is sometimes beneficial to use a point of compromise between $\hat{\boldsymbol{\Omega}}^{(0)}$ and $\hat{\boldsymbol{\Omega}}^{(1)}$ instead of the MLE like SNB does. Also, we discuss why the $L_{1} / L_{2}-\mathrm{NB}$ approach (including the adaptive version) can discard only irrelevant predictors and not redundant predictors.

First, we define the redundancy and irrelevance concepts and briefly discuss their effect on the NB model. We define a predictor as noisy if it is irrelevant for the class variable or is redundant to another predictor. Similar definitions of irrelevance and redundancy can be found, for example, in Kohavi and John [12] and Langley and Sage [13].

A discrete predictor $X_{i}$ is irrelevant for $Y$ if

$$
\begin{aligned}
& P\left(Y=j \mid X_{i}=k\right)=P(Y=j), \quad \forall k \in\left\{1, \ldots, M_{i}\right\}, \\
& \quad \forall j \in\{1, \ldots, J\},
\end{aligned}
$$

so that the value of $X_{i}$ does not give any information about the value of $Y$. Equivalently, we can say that the within-class parameters of predictor $X_{i}$ are equal for all class values. The definition for a continuous predictor is analogous.

On the other hand, two predictors $X_{i_{1}}$ and $X_{i_{2}}$ are redundant when there is a dependency between them.

Let $H(\cdot)$ represent the entropy function. Two predictors $X_{i_{1}}$ and $X_{i_{2}}$ are fully redundant when

$H\left(X_{i_{1}} \mid X_{i_{2}}\right)=H\left(X_{i_{2}} \mid X_{i_{1}}\right)=0$.

On the other hand, they are completely independent when

$H\left(X_{i_{1}} \mid X_{i_{2}}\right)=H\left(X_{i_{1}}\right), \quad H\left(X_{i_{2}} \mid X_{i_{1}}\right)=H\left(X_{i_{2}}\right)$. 


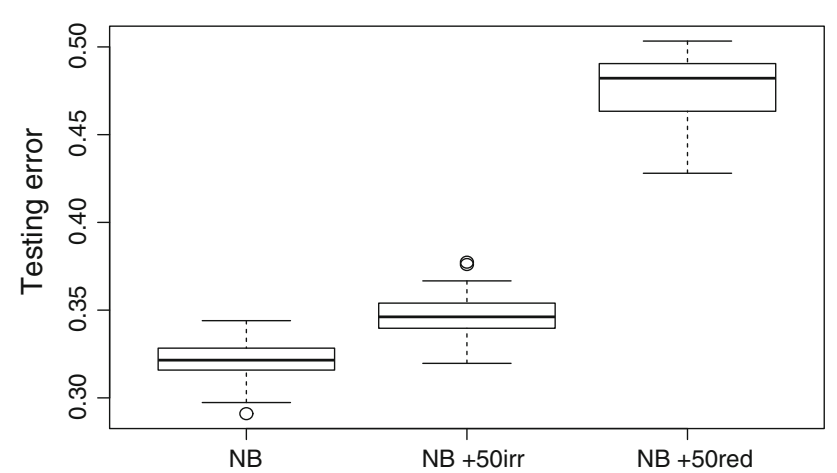

Fig. 1 Boxplots for the testing errors of NB without noisy variables (left), NB with 50 irrelevant predictors and NB with 50 redundant predictors

Note that these conditions are just extremes of a continuum. In real-world data, predictors are rarely fully redundant or completely independent. Instead, they typically are somewhere between these two extreme conditions.

When $N \rightarrow \infty$ and $p$ is finite (i.e., the complete information case), irrelevant variables do not increment the expected error of a NB classifier because $\hat{\boldsymbol{\Omega}}^{(1)}=\hat{\mathbf{\Omega}}^{(0)}$ holds exactly. In the realistic case, when $N$ is finite, we only have $\hat{\boldsymbol{\Omega}}^{(1)} \simeq \hat{\mathbf{\Omega}}^{(0)}$. In the presence of many irrelevant predictors, these small differences accumulate and can finally bias the actual decision and degrade the classification accuracy.

It is well known, however, that, for NB, redundant predictors have a more harmful effect than irrelevant predictors [5]. Figure 1 shows testing errors of NB models obtained from three different types of discrete synthetic data sets. The first type has three non-noisy predictors, $\left\{X_{1}, X_{2}, X_{3}\right\}$, that are generated from the following probabilities

$$
\begin{aligned}
& \boldsymbol{\Theta}_{1}=\left(\begin{array}{lll}
0.80 & 0.33 & 0.33 \\
0.10 & 0.33 & 0.33 \\
0.10 & 0.33 & 0.33
\end{array}\right), \\
& \boldsymbol{\Theta}_{2}=\boldsymbol{\Theta}_{3}=\left(\begin{array}{lll}
0.33 & 0.30 & 0.30 \\
0.33 & 0.10 & 0.60 \\
0.33 & 0.60 & 0.10
\end{array}\right),
\end{aligned}
$$

so that predictor $X_{1}$ discriminates between the first and the other two class values, and predictors $X_{2}$ and $X_{3}$ mainly discriminate between the second and third class values; $\pi$ is defined as being equal for all three class values. The other two types have, in addition, 50 irrelevant discrete predictors and 50 (fully) redundant discrete predictors, respectively. The class can take three values, each with the same frequency. We have conducted 100 experiments, generating training data sets with $N=1,000$ instances and test data sets with $N_{\text {te }}=$ 3,000 instances. Notice that both kinds of noisy predictors, but especially the redundant ones, decrease accuracy.

Using the same data, Fig. 2 illustrates, for one experiment, the evolution of the testing error for an increasing number
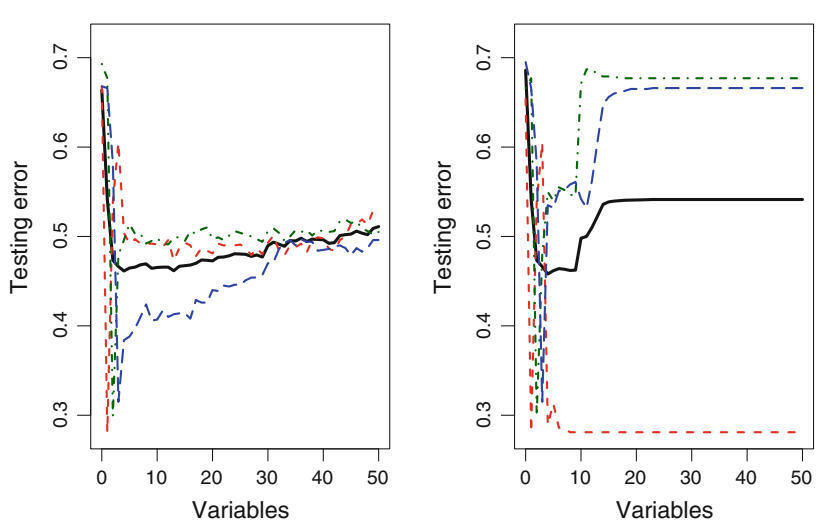

Fig. 2 Evolution of the testing error for an increasing number of irrelevant (left) and redundant (right) predictors. The first three predictors are non-noisy

predictors. The $X$-axis represents the number of predictors in the model. The first three added predictors (leftmost part of the graphs) are relevant, and the others, up to 50, are irrelevant (left hand graph) or redundant (right hand graph). Predictors are redundant with regard to the first non-noisy predictor. The solid line represents the error computed on the complete testing data set, whereas the other lines represent the error for each of the three class values. The black line represents the mean of the other three lines. We find that the class value that is best discriminated by the first predictor (short-dashed line) decreases the error in the presence of redundant predictors, but the other class values are no longer distinguishable. Irrelevant predictors, on the other hand, produce a more uniform and moderate increment of the error.

Ideally, SNB only adds predictors that reduce the classification error to the model. Hence, it will discard both redundant and irrelevant predictors, and retain those variables that are relevant but not redundant. However, as mentioned above, relevance and redundancy are not absolute concepts. What will SNB do with a set of relevant but non-fully redundant predictors? Let us suppose that there are two predictors, $X_{i_{1}}$ and $X_{i_{2}}$, that are (non-fully) redundant, and each carries valuable information. In this paper, we claim that a NB model that balances the contribution of these predictors may be better than a classic NB model that either excludes or fully includes them, like SNB does.

We use an example to illustrate this point. Let us first define

$\boldsymbol{\Theta}_{i}^{\left(\alpha_{i}\right)}=\alpha_{i} \boldsymbol{\Theta}_{i}^{(1)}+\left(1-\alpha_{i}\right) \boldsymbol{\Theta}_{i}^{(0)}$,

$\boldsymbol{\mu}_{i}^{\left(\alpha_{i}\right)}=\alpha_{i} \boldsymbol{\mu}_{i}^{(1)}+\left(1-\alpha_{i}\right) \boldsymbol{\mu}_{i}^{(0)}$,

$\sigma_{i}^{\left(\alpha_{i}\right)}=\alpha_{i} \sigma_{i}^{(1)}+\left(1-\alpha_{i}\right) \sigma_{i}^{(0)}$,

where, $\alpha_{i} \in[0,1]$. Hence, $\hat{\boldsymbol{\Omega}}_{i}^{\left(\alpha_{i}\right)}$ is a linear combination of $\hat{\boldsymbol{\Omega}}_{i}^{(0)}$ and $\hat{\boldsymbol{\Omega}}_{i}^{(1)}$, where $\alpha_{i}$ refers to predictor $X_{i}$. Within this notation, we can say that SNB only considers values 

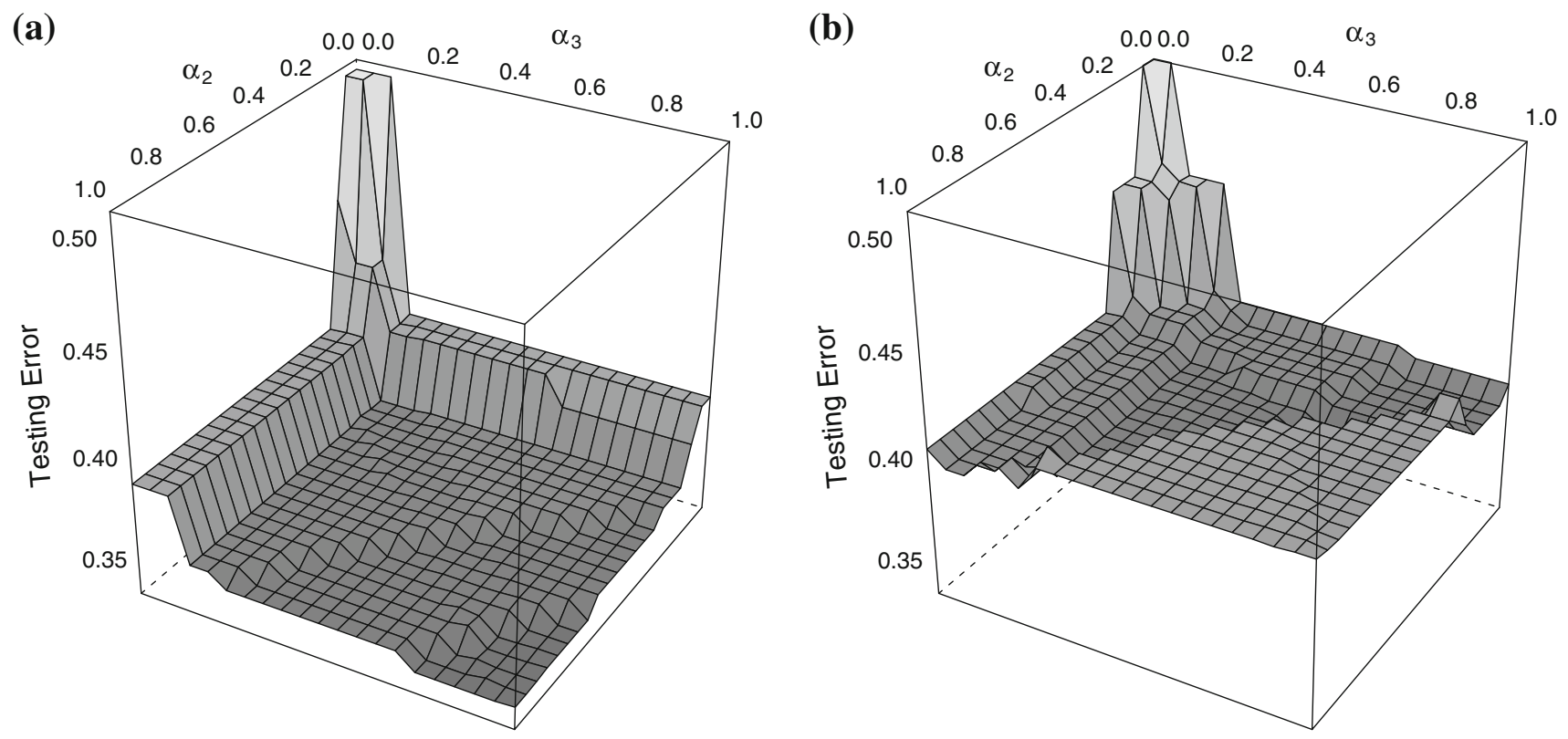

Fig. 3 a Testing error when discrete predictors $X_{2}$ and $X_{3}$ are not made redundant, $\mathbf{b}$ testing error when predictors are somewhat redundant

$\alpha_{i} \in\{0,1\}$ (exclusion or inclusion, respectively, of predictor $X_{i}$ ).

Now, we consider a training data set with $N=1,000$ instances and a testing data set with $N_{\text {te }}=3,000$ instances, with three predictors whose CPTs are given in (12). Now, we consider making $X_{2}$ and $X_{3}$ redundant by setting $x_{r 2}=x_{r 3}$ for some proportion of the data instances.

Let us consider NB models with parameters $\hat{\boldsymbol{\Theta}}_{1}^{(1)}, \hat{\boldsymbol{\Theta}}_{2}^{\left(\alpha_{2}\right)}$ and $\hat{\boldsymbol{\Theta}}_{3}^{\left(\alpha_{3}\right)}$. For a grid of values $\alpha_{2}, \alpha_{3} \in[0,1]$, Fig. 3a shows testing errors when $X_{2}$ and $X_{3}$ are not made redundant, that is, if we have not set $x_{r 2}=x_{r 3}$ at any time.

Figure $3 \mathrm{~b}$ shows testing errors when $X_{2}$ and $X_{3}$ are somewhat redundant, that is, after setting $x_{r 2}=x_{r 3}$ for some proportion of the data instances.

We find that, when $X_{2}$ and $X_{3}$ are independent, the minimum error is achieved when $\alpha_{2}, \alpha_{3}$ are equal to 1, i.e., when $\hat{\boldsymbol{\Theta}}_{2}=\hat{\boldsymbol{\Theta}}_{2}^{(1)}$ and $\hat{\boldsymbol{\Theta}}_{3}=\hat{\boldsymbol{\Theta}}_{3}^{(1)}$. On the other hand, when there is some dependence between $X_{2}$ and $X_{3}$, and $X_{1}$ is already part of the model, the best model is somewhere in $0<\alpha_{2}, \alpha_{3}<1$.

Figure 4 illustrates the same scenario for continuous predictors. Figure 4a shows testing errors when $X_{2}$ and $X_{3}$ are independent, and Fig. $4 \mathrm{~b}$ shows testing errors when $X_{2}$ and $X_{3}$ are somewhat redundant.

Although the effect is less obvious than in the discrete case, the conclusion is analogous.

These examples illustrate that it may be worthwhile finding a tradeoff between the MLE $\left(\hat{\boldsymbol{\Omega}}_{i}^{(1)}\right)$ and the parameters that remove the predictor from the model $\left(\hat{\boldsymbol{\Omega}}_{i}^{(0)}\right)$. This is the main motivation for proposing the forward stagewise naïve Bayes approach.
Finally, we note that, although $L_{1} / L_{2}-\mathrm{NB}$ is a natural choice for applying regularization to the NB model, it discards only irrelevant and not redundant predictors. It discards irrelevant predictors because, since $\hat{\boldsymbol{\Omega}}_{i}^{(0)}$ is not very different from $\hat{\boldsymbol{\Omega}}_{i}^{(1)}$ in this case, they make only a small contribution to the loss function in optimization problems (8) and (9). Note, however, that setting $\hat{\boldsymbol{\Omega}}_{i}=\hat{\mathbf{\Omega}}_{i}^{(0)}$ amounts to removing this predictor from the NB model, but it does not lead to the exclusion of the predictor from the loss function calculation in the optimization problem. In other words, according to this formulation, all predictors participate in the loss function (Eq. (1)), even when they can be simplified from the classification rule (Eqs. (4) and (5)). Therefore, if, for example, two predictors are fully redundant but separately relevant, the $L_{1} / L_{2}$-NB (or a $L_{1} / L_{2}-\mathrm{NB}$ ) approach will add them both to the model, because, according to the log-likelihood formulation, both have a relevant impact on the loss function, no matter what the state of the other is. In other words, the inclusion of one predictor does not change the effect of the other on the loss function. In general terms, any algorithm that solves optimization problems (8) or (9) will select either both predictors or neither.

\section{Forward stagewise naïve Bayes}

We now introduce a more cautious version of the SNB approach, the forward stagewise naïve Bayes (fsNB). Like $\mathrm{SNB}$, fsNB is a greedy algorithm but, instead of moving a set of parameters from $\hat{\mathbf{\Omega}}_{i}^{(0)}$ to $\hat{\mathbf{\Omega}}_{i}^{(1)}$ at each iteration, it takes small steps from $\hat{\boldsymbol{\Omega}}_{i}^{\left(\alpha_{i}\right)}$ to $\hat{\boldsymbol{\Omega}}_{i}^{\left(\alpha_{i}+\epsilon\right)}$, where $\epsilon>0$ is some small 

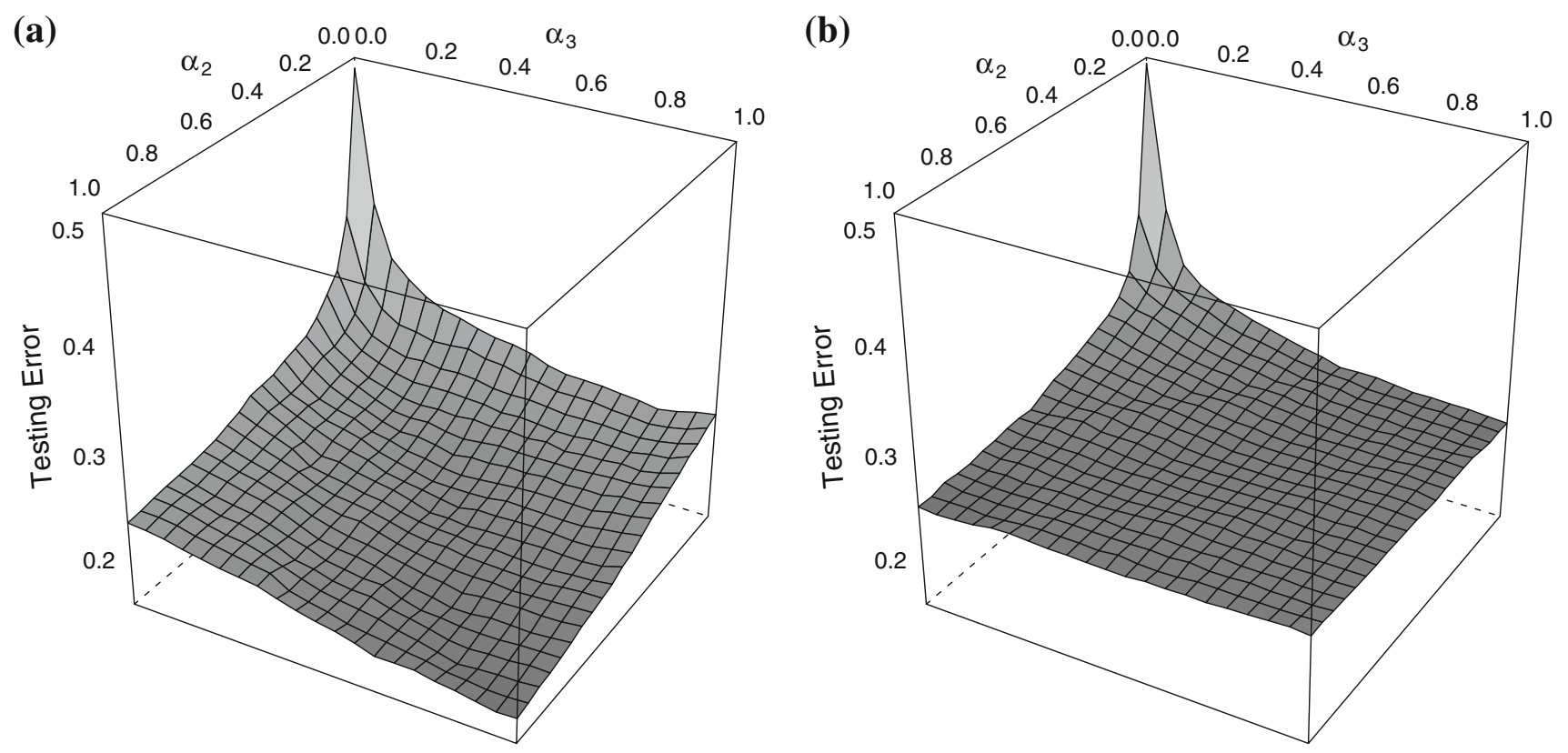

Fig. 4 a Testing error when continuous predictors $X_{2}$ and $X_{3}$ are not made redundant, $\mathbf{b}$ testing error when predictors are somewhat redundant

constant and $\alpha_{i}$ determines the current parameters of predictor $X_{i}$ (see Eq. (13)). We can informally say that fsNB is to SNB what stagewise regression is to stepwise regression [17].

The rationale of this approach is to deal with the situation discussed in Sect. 4, i.e., when there are partially redundant variables that each carry separate information. By giving a balanced estimation of their parameters, we expect to retain the valuable information while minimizing the harmful effect of redundancy.

Concerning the greedy strategy, there is one important matter to address. At each iteration, we need to evaluate each predictor so as to decide which is going to be adjusted. There are two simple strategies for finding which predictor is most worth updating. Let us suppose that the parameters of predictor $X_{i}$ are $\hat{\mathbf{\Omega}}_{i}^{\left(\alpha_{i}\right)}$. The first strategy is to evaluate predictor $X_{i}$ by checking $\hat{\mathbf{\Omega}}_{i}^{\left(\alpha_{i}+\epsilon\right)}$. The second strategy is to check $\hat{\mathbf{\Omega}}_{i}^{(1)}$. Whatever we do, the predictor that leads to the greatest error decrement will be updated by $\epsilon$ (and the others are unchanged). Neither approach is problem-free. In the first case, it is often not possible to decide how important a predictor is by just looking at some small increment $\epsilon$.

In the second case, if we look at the complete update of the parameters of the predictor, the contribution of other predictors with low $\alpha_{i^{\prime}}\left(i^{\prime} \neq i\right)$ could become negligible. Even when predictor $X_{i}$ is important, the model accuracy may decrease considerably if the contribution of other important variables (almost) disappears.

Figure 5 illustrates this situation for two predictors, one relevant (left) and one irrelevant (right). It shows, at some early step of the algorithm, the evolution of the training and
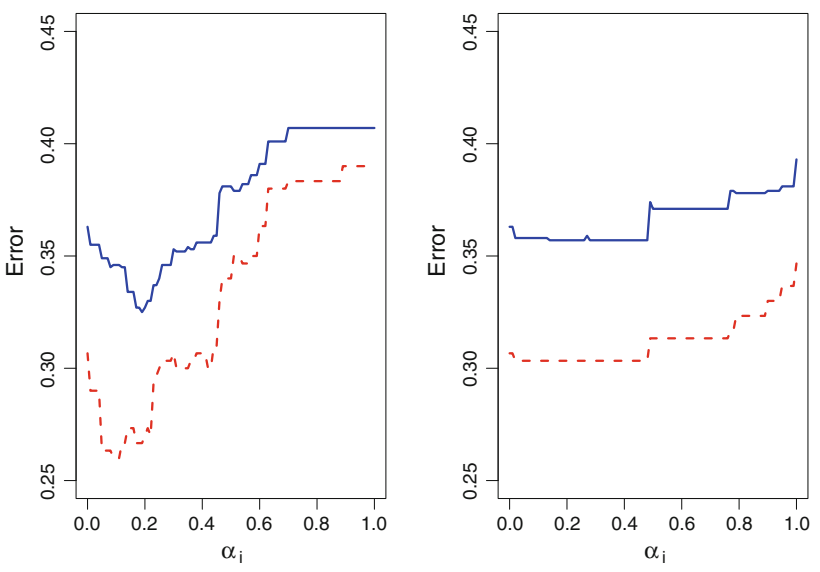

Fig. 5 Training error (dashed line) and testing error (solid line) across the evolution of two variables, one relevant (left) and one irrelevant (right)

testing errors when we increase $\alpha_{i}$ for each predictor. Note that, in order to select the relevant rather than the irrelevant predictor, we have to look at a point between $\hat{\mathbf{\Omega}}^{\left(\alpha_{i}+\epsilon\right)}$ and $\hat{\mathbf{\Omega}}^{(1)}$, where the training (and testing error) is most decreased.

To do this, we consider some further steps $v$ at each iteration, i.e., for each predictor, we check the error for $\hat{\mathbf{\Omega}}_{i}^{\alpha_{i}+\epsilon}, \hat{\mathbf{\Omega}}_{i}^{\alpha_{i}+2 \epsilon}, \ldots, \hat{\boldsymbol{\Omega}}_{i}^{\alpha_{i}+t \epsilon}, \ldots, \hat{\mathbf{\Omega}}_{i}^{\alpha_{i}+\nu \epsilon}$. This way, at each iteration, we select the optimal values $\{i, t\}$, and update the parameters accordingly. Parameters $\epsilon$ and $v$ define how detailed is the search at each step and may have an impact in the computational efficiency of the algorithm. Reasonable variations of them, however, does not greatly change the algorithm accuracy. 


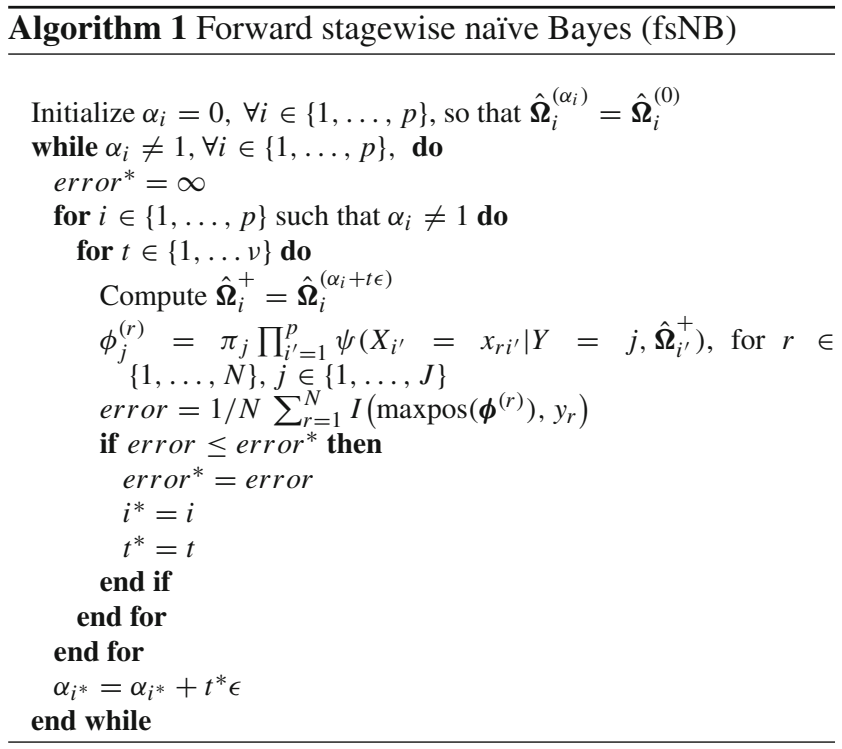

Algorithm 1 details the fsNB method in pseudocode format. The main part consists of two nested loops that look for the best pair $\{i, t\}$ at each iteration. Like SNB, the fitting criterion is the training error. The function $I(\cdot, \cdot)$ is an indicator function that outputs 1 if its arguments are equal and 0 if otherwise.

To minimize the computational cost, we can stop the procedure early if the training error has not improved during a certain number of iterations. We have observed that the minimum testing error is very rarely found after the training error comes to a standstill, which makes this strategy promising.

\section{Model selection}

Both the $L_{1} / L_{2}$-NB (using a grid of $\lambda$ values) and the fsNB approaches generate a potentially large set of models, from which a final model needs to be selected. We can use a validation subset of the data set (if data are abundant), $K$-fold crossvalidation, or some penalized criterion, which is typically the training loss plus some estimation of the optimism of the training loss rate. In this paper, we use the AIC statistic [1]: $\mathrm{AIC}=Q(\boldsymbol{D}, \hat{\boldsymbol{\Omega}})+\frac{2}{N} d$,

where the loss function is the mean cross-entropy or deviance

$$
\begin{aligned}
& Q(\boldsymbol{D}, \hat{\mathbf{\Omega}})=\frac{1}{N} \sum_{r=1}^{N} \\
& -2 \log \left(Y=y_{r} \mid X_{1}=x_{r 1}, \ldots, X_{p}=x_{r p}, \hat{\boldsymbol{\Omega}}, \hat{\boldsymbol{\pi}}\right),
\end{aligned}
$$

and $d$ represents the degrees of freedom of the model, which we compute as

$d=\sum_{i=1}^{p} I\left(\alpha_{i}>0\right)$,
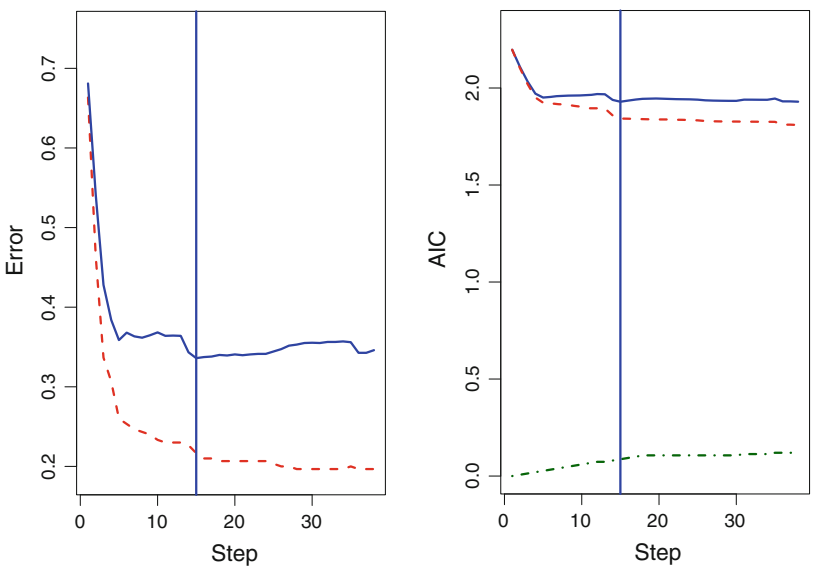

Fig. 6 Left Training error (dashed line) and testing error (solid line). Minimum testing error step is highlighted with a vertical line. Right AIC statistic (solid line), loss function (dashed line) and AIC penalty term (dashed-dotted line). The step with the lowest AIC statistic value is highlighted with a vertical line.

where $I(\cdot)$ outputs 1 if the argument is true and 0 if otherwise. Since a NB model is linear and $0<d \leq p$, this is a reasonable estimation.

For $L_{1} / L_{2}-\mathrm{NB}$, a possible natural choice, instead of Eq. (14), for computing $d$, in the discrete and continuous case, respectively, would be

$d=\sum_{i=1}^{p} \frac{1}{J M_{i}} \sum_{j=1}^{J} \sum_{k=1}^{M_{i}} \frac{\theta_{i k j}-\hat{\theta}_{i j k}^{(0)}}{\hat{\theta}_{i j k}^{(1)}-\hat{\theta}_{i j k}^{(0)}}$,

$d=\sum_{i=1}^{p} \frac{1}{J} \sum_{j=1}^{J}\left(\frac{\mu_{i j}-\hat{\mu}_{i j}^{(0)}}{\hat{\mu}_{i j}^{(1)}-\hat{\mu}_{i j}^{(0)}}+\frac{\sigma_{i j}-\hat{\sigma}_{i j}^{(0)}}{\hat{\sigma}_{i j}^{(1)}-\hat{\sigma}_{i j}^{(0)}}\right)$.

For fsNB, this would simplify to

$d=\sum_{i=1}^{p} \alpha_{i}$.

We have found, however, that the results are better using Eq. (14). Therefore, in this paper, we compute $d$ using Eq. (14).

Figure 6 shows, for some generated data set with three relevant variables and twelve irrelevant variables, training and testing errors (left) and the AIC statistic, loss function and AIC penalty term (right) for a sequence of NB models generated by fsNB. Note that, in this example, the best model is nearly the same for the test data as for AIC.

\section{Experiments}

So far, we have presented some examples to illustrate the claims. In this section, we perform a more systematic 
evaluation of the methods. We test the methods first on some synthetic data sets and then on some data sets derived from the Diabetes data set, taken from the UCI repository ${ }^{1}$.

\subsection{Synthetic data sets}

We now run the algorithms on a number of synthetic training/test data sets, generated from several scenarios. Each data set has $p=20$ predictors, which can be discrete $\left(M_{i}=3\right)$ or continuous. Training data sets have $N=300$ instances and test data sets have $N_{\text {te }}=3,000$ instances. There are $J=3$ class values.

Within each data set, there are $p_{1}=3$ non-noisy predictors, $p_{2}=7$ non-fully redundant predictors, which nevertheless carry some information, and $p_{3}=10$ totally noisy predictors, which may be irrelevant or redundant to any of the $p_{1}$ non-noisy predictors. We call these three groups, respectively, $V_{1}, V_{2}$ and $V_{3}$. Hence, $p=p_{1}+p_{2}+p_{3}$.

For each experiment, we randomly generate the "true" parameters that produce the data as follows. In the discrete case, for each predictor in $V_{1}$, we sample

$\boldsymbol{\theta}_{i j} \sim \operatorname{Dir}(\boldsymbol{c})$,

where $\boldsymbol{\theta}_{i j}$ is the $j$ th column of $\boldsymbol{\Theta}_{i}$ and $\operatorname{Dir}(\boldsymbol{c})$ is a Dirichlet distribution with the vector of shape parameters $c$, whose components are all equal except one, which is different for each $j \in\{1, \ldots, J\}$.

Within each data set, all predictors in $V_{2}$ have the same CPT, which is similarly generated from a Dirichlet distribution. Each predictor in $V_{2}$ is slightly redundant to the preceding and following predictor, i.e., $H\left(X_{i} \mid X_{i-1}\right)<H\left(X_{i}\right)$ and $H\left(X_{i} \mid X_{i+1}\right)<H\left(X_{i}\right)$ for $i \in\left\{p_{1}+2, p_{1}+p_{2}-1\right\}$ (assuming that predictors in $V_{2}$ are preceded by predictors in $V_{1}$ and followed by predictors in $V_{3}$ ). This redundancy is achieved by setting the value of the predictor $X_{i}$ to be equal to either $X_{i-1}$ or $X_{i+1}$ with a probability equal to 0.5 .

If predictors in $V_{3}$ are irrelevant, they have the parameters of a multinomial distribution, and they are generated from a Dirichlet distribution with equal hyperparameters. In other words, the CPT columns of each predictor in $V_{3}$ are all equal. If predictors in $V_{3}$ are redundant, the parameters are generated as for irrelevant predictors. In this case, however, each predictor has a very low conditional entropy given some randomly selected predictor from $V_{1}$. This is achieved by setting the value of the predictor in $V_{3}$ to be equal to the predictor in $V_{1}$ with a probability equal to 0.9 . Note that, once the corresponding predictor in $V_{1}$ has been added, this predictor does not carry any additional useful information at all.

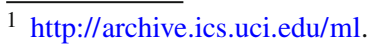

In the continuous case, predictors are generated from Gaussian distributions. For each predictor in $V_{1}$, we sample $\mu_{i j} \sim \operatorname{Unif}(-2,2), \quad \sigma_{i j}=0.75$,

where Unif $(-2,2)$ is the uniform distribution between -2 and 2 .

As in the discrete case, all predictors in $V_{2}$ have the same parameters. Again, let $X_{i}$ be equal to either $X_{i-1}$ or $X_{i+1}$ with a probability equal to 0.5 .

If predictors in $V_{3}$ are irrelevant, we have $\mu_{i j}=m_{i}$, for all $j \in\{1, \ldots, C\}$. The value $m_{i}$ is generated from a uniform distribution in the interval $(-2,2)$. If predictors in $V_{3}$ are redundant, parameters are generated similarly, but, for each data instance, each predictor in $V_{3}$ is bound, with a probability equal to 0.9 , to have the same value as some predictor in $V_{1}$, plus some small noise.

Finally, we set $\pi=(1 / 3,1 / 3,1 / 3)$ in all cases. Hence, we have four different scenarios, which are the four possible combinations of discrete/continuous predictors and irrelevant/redundant predictors within $V_{3}$.

We generate 100 different data sets from each scenario using the Bayes rule (taking into account the mentioned redundancies). Table 1 shows, for each data set type, the means and standard deviations of the testing misclassification error, number of selected variables and number of (fully) noisy-selected variables, for $\mathrm{NB}, \mathrm{SNB}, \mathrm{WNB}, \mathrm{a} L_{1} / L_{2}-\mathrm{NB}$ and fsNB. We have run fsNB with parameters $\epsilon=0.025, v=$ 20, which we have empirically observed to be a good choice in general. Also, we use early stopping (see Sect. 5). For comparison's sake, we have also run NB on a subset of predictors, selected by (prefiltering) correlation-based feature selection [10]. We denote this approach as CFS + NB.

We find that there are two clearly different scenarios. First, when the noisy predictors are irrelevant, the methods that do not select variables (NB and WNB) perform best. This is certainly expectable, because, as discussed in Sect. 4, NB is relatively robust to irrelevant predictors, and there are not enough to significantly reduce accuracy. Note, however, that, in the discrete case at least, fsNB is closer to NB and WNB than the other wrapper selective methods and also than $\mathrm{CFS}+\mathrm{NB}$.

Second, when the noisy predictors are redundant, fsNB beats the others. CFS + NB also works fine and turns out to be the most accurate method in the continuous case. The differences between fsNB and SNB are probably due to the fsNB's balanced estimation of parameters of the predictors in $V_{2}$. The number of selected predictors is not very different for fsNB and SNB in this case. CFS + NB clearly selects more predictors than the wrapper approaches. Finally, note that, excepting for the continuous with irrelevant noise variables data set, a $L_{1} / L_{2}-\mathrm{NB}$ does not excel. Although $L_{1} / L_{2}-\mathrm{NB}$ is not shown in Table $1, \mathrm{a} L_{1} / L_{2}-\mathrm{NB}$ is slightly better than its nonadaptive counterpart. Regarding computational cost, SNB 
Table 1 Mean testing misclassification error (top), mean number of selected variables (middle) and mean number of (fully) noisy selected variables (bottom) for each synthetic data set type and each method

\begin{tabular}{|c|c|c|c|c|c|c|}
\hline Data set type & NB & SNB & WNB & $\mathrm{a} L_{1} / L_{2}-\mathrm{NB}$ & fsNB & $\mathrm{CFS}+\mathrm{NB}$ \\
\hline \multicolumn{7}{|c|}{ Misclassification error } \\
\hline DI & $0.076( \pm 0.03)$ & $0.080( \pm 0.03)$ & $\mathbf{0 . 0 7 5}( \pm 0.03)$ & $0.079( \pm 0.06)$ & $0.077( \pm 0.02)$ & $0.166( \pm 0.18)$ \\
\hline CI & $\mathbf{0 . 0 8 2}( \pm 0.04)$ & $0.083( \pm 0.05)$ & $\mathbf{0 . 0 8 2}( \pm 0.04)$ & $0.083( \pm 0.05)$ & $0.083( \pm 0.05)$ & $0.087( \pm 0.02)$ \\
\hline DR & $0.152( \pm 0.07)$ & $0.076( \pm 0.02)$ & $0.131( \pm 0.08)$ & $0.171( \pm 0.10)$ & $\mathbf{0 . 0 7 0}( \pm 0.03)$ & $0.082( \pm 0.06)$ \\
\hline CR & $0.132( \pm 0.05)$ & $0.097( \pm 0.03)$ & $0.132( \pm 0.05)$ & $0.158( \pm 0.10)$ & $0.090( \pm 0.03)$ & $\mathbf{0 . 0 8 3}( \pm 0.08)$ \\
\hline \multicolumn{7}{|c|}{ Number of selected variables } \\
\hline DI & - & $6.2( \pm 1.4)$ & - & $6.5( \pm 2.1)$ & $6.3( \pm 1.7)$ & $10.8( \pm 0.8)$ \\
\hline $\mathrm{CI}$ & - & $6.0( \pm 1.5)$ & - & $6.0( \pm 1.2)$ & $\mathbf{5 . 3}( \pm 1.8)$ & $10.0( \pm 0.1)$ \\
\hline DR & - & $5.7( \pm 1.5)$ & - & $10.2( \pm 2.1)$ & $\mathbf{5 . 6}( \pm 1.7)$ & $10.4( \pm 0.6)$ \\
\hline $\mathrm{CR}$ & - & $\mathbf{5 . 5 ^ { * }}( \pm 1.3)$ & - & $11.8( \pm 1.9)$ & $6.6( \pm 2.3)$ & $10.8( \pm 1.0)$ \\
\hline \multicolumn{7}{|c|}{ Number of noisy selected variables } \\
\hline DI & - & $0.6( \pm 0.8)$ & - & $\mathbf{0 . 2}( \pm 0.7)$ & $0.3( \pm 0.9)$ & $0.8( \pm 0.9)$ \\
\hline $\mathrm{CI}$ & - & $0.8( \pm 0.8)$ & - & $\mathbf{0 . 1}( \pm 0.3)$ & $\mathbf{0 . 1}( \pm 0.2)$ & $0.4( \pm 0.5)$ \\
\hline DR & - & $0.4( \pm 0.7)$ & - & $5.1( \pm 0.8)$ & $\mathbf{0 . 2}( \pm 0.5)$ & $0.6( \pm 0.6)$ \\
\hline $\mathrm{CR}$ & - & $\mathbf{0 . 8}( \pm 0.4)$ & - & $5.9( \pm 0.8)$ & $1.1( \pm 0.7)$ & $1.0( \pm 1.0)$ \\
\hline
\end{tabular}

Data set types are discrete with irrelevant noise variables (DI), continuous with irrelevant noise variables (CI), discrete with redundant noise variables (DR) and continuous with redundant noise variables (CR). The best result for each row is highlighted in bold. NB and WNB have been omitted from the variable selection report because they do not perform variable selection

Table 2 Mean 10-CV cross-validated misclassification error (top) and number of selected variables (bottom) for each data set derived from the Diabetes data set and each method

\begin{tabular}{|c|c|c|c|c|c|c|}
\hline Data set & NB & SNB & WNB & $\mathrm{a} L_{1} / L_{2}-\mathrm{NB}$ & fsNB & $\mathrm{CFS}+\mathrm{NB}$ \\
\hline \multicolumn{7}{|c|}{ Misclassification error } \\
\hline$\pi=(1 / 4,3 / 4)$ & $0.28( \pm 0.06)$ & $0.23( \pm 0.05)$ & $0.28( \pm 0.06)$ & $0.25( \pm 0.07)$ & $\mathbf{0 . 2 1}( \pm 0.06)$ & $0.26( \pm 0.10)$ \\
\hline$\pi=(1 / 2,1 / 2)$ & $0.28( \pm 0.07)$ & $0.27( \pm 0.06)$ & $0.28( \pm 0.07)$ & $0.27( \pm 0.08)$ & $\mathbf{0 . 2 6}( \pm 0.07)$ & $0.27( \pm 0.15)$ \\
\hline$\pi=(3 / 4,1 / 4)$ & $0.20( \pm 0.04)$ & $0.18( \pm 0.05)$ & $0.20( \pm 0.04)$ & $0.19( \pm 0.05)$ & $\mathbf{0 . 1 6}( \pm 0.06)$ & $0.18( \pm 0.15)$ \\
\hline \multicolumn{7}{|c|}{ Number of selected predictors } \\
\hline$\pi=(1 / 4,3 / 4)$ & - & $1.7( \pm 0.48)$ & - & $4.4( \pm 3.80)$ & $2.2( \pm 0.42)$ & $3.0( \pm 0.42)$ \\
\hline$\pi=(1 / 2,1 / 2)$ & - & $\mathbf{3 . 3}( \pm 0.48)$ & - & $8.3( \pm 0.48)$ & $4.4( \pm 0.95)$ & $4.2( \pm 0.51)$ \\
\hline$\pi=(3 / 4,1 / 4)$ & - & $3.3( \pm 0.67)$ & - & $8.4( \pm 0.84)$ & $2.4( \pm 0.51)$ & $4.9( \pm 0.78)$ \\
\hline
\end{tabular}

The best result for each row is highlighted in bold. NB and WNB have been omitted from the variable selection report because they do not perform variable selection

and fsNB take, respectively, 125.10 and 6732.25 evaluations on average. The computational cost is similar for all data sets.

\subsection{Diabetes data sets}

We now carry out some experiments with real data. We use the Diabetes data set, which has $N=442$ instances and $p=10$ continuous predictors. Although the response is continuous, we generate data sets for binary classification by means of the rule

$y_{r}= \begin{cases}0 & \text { if } \tilde{y}_{r}<\tau \\ 1 & \text { if } \tilde{y}_{r} \geq \tau .\end{cases}$ where $\tilde{y}_{r}$ is the continuous response and $\tau$ is some real constant. We generate three different data sets by setting $\tau$ to be equal to the first three quartiles. Therefore, for each data set, we have, respectively, $\pi=(1 / 4,3 / 4), \pi=(1 / 2,1 / 2)$ and $\pi=(3 / 4,1 / 4)$.

Table 2 illustrates the results obtained from 10-fold crossvalidation, which include testing misclassification error and number of selected variables. As before, the tested methods are NB, SNB, WNB, a $L_{1} / L_{2}-\mathrm{NB}$, fsNB and CFS + NB. We have run fsNB with parameters $\epsilon=0.025, v=20$, using early stopping.

Note that fsNB is the most accurate, followed by SNB and $\mathrm{CFS}+\mathrm{NB}$. 
Table 3 Mean 10-CV cross-validated misclassification error (top) and number of selected variables (bottom) for each subject in the Starplus data set and each method

\begin{tabular}{|c|c|c|c|c|c|c|}
\hline Subject & NB & SNB & WNB & $\mathrm{a} L_{1} / L_{2}-\mathrm{NB}$ & fsNB & $\mathrm{CFS}+\mathrm{NB}$ \\
\hline \multicolumn{7}{|c|}{ Misclassification error } \\
\hline 04799 & $0.47( \pm 0.08)$ & $0.45( \pm 0.04)$ & $0.47( \pm 0.08)$ & $0.52( \pm 0.07)$ & $\mathbf{0 . 4 1}( \pm 0.26)$ & $0.50( \pm 0.23)$ \\
\hline 05675 & $0.44( \pm 0.07)$ & $\mathbf{0 . 4 3}( \pm 0.06)$ & $0.44( \pm 0.07)$ & $0.51( \pm 0.06)$ & $0.50( \pm 0.11)$ & $0.46( \pm 0.19)$ \\
\hline 04820 & $0.44( \pm 0.07)$ & $0.43( \pm 0.06)$ & $0.44( \pm 0.07)$ & $0.55( \pm 0.03)$ & $0.37( \pm 0.34)$ & $\mathbf{0 . 3 4}( \pm 0.21)$ \\
\hline 05680 & $0.45( \pm 0.05)$ & $0.44( \pm 0.06)$ & $0.45( \pm 0.05)$ & $0.57( \pm 0.04)$ & $\mathbf{0 . 3 5}( \pm 0.26)$ & $0.48( \pm 0.16)$ \\
\hline 04847 & $0.36( \pm 0.06)$ & $\mathbf{0 . 3 3}( \pm 0.05)$ & $0.36( \pm 0.06)$ & $0.57( \pm 0.06)$ & $0.35( \pm 0.06)$ & $0.44( \pm 0.18)$ \\
\hline 05710 & $0.40( \pm 0.07)$ & $0.45( \pm 0.06)$ & $0.40( \pm 0.07)$ & $0.55( \pm 0.02)$ & $\mathbf{0 . 3 6}( \pm 0.26)$ & $0.48( \pm 0.32)$ \\
\hline \multicolumn{7}{|c|}{ Number of selected predictors } \\
\hline 04799 & - & $3.8( \pm 1.51)$ & - & $\mathbf{0 . 2}( \pm 0.02)$ & $1.0( \pm 0.77)$ & $1.1( \pm 0.81)$ \\
\hline 05675 & - & $3.5( \pm 1.32)$ & - & $\mathbf{0 . 3}( \pm 0.01)$ & $0.9( \pm 0.30)$ & $1.0( \pm 0.66)$ \\
\hline 04820 & - & $4.9( \pm 1.31)$ & - & $\mathbf{0 . 1}( \pm 0.01)$ & $1.3( \pm 1.04)$ & $0.9( \pm 1.01)$ \\
\hline 05680 & - & $5.5( \pm 2.50)$ & - & $\mathbf{0 . 3}( \pm 0.02)$ & $1.2( \pm 0.79)$ & $1.2( \pm 0.36)$ \\
\hline 04847 & - & $4.2( \pm 1.51)$ & - & $\mathbf{0 . 1}( \pm 0.02)$ & $1.8( \pm 0.03)$ & $2.1( \pm 0.77)$ \\
\hline 05710 & - & $5.9( \pm 1.82)$ & - & $\mathbf{0 . 2}( \pm 0.03)$ & $1.5( \pm 0.91)$ & $2.0( \pm 0.52)$ \\
\hline
\end{tabular}

The best result for each row is highlighted in bold. NB and WNB have been omitted from the variable selection report because they do not perform variable selection

Note that $L_{1} / L_{2}-\mathrm{NB}$ is always worse than fsNB and $\mathrm{SNB}$, which is a possible sign of certain redundancy among the predictors (that a $L_{1} / L_{2}-\mathrm{NB}$ is not purging). In these data sets, WNB obtains very similar results to NB. None of the methods, however, is very accurate when $\pi=(1 / 4,3 / 4)$. In this case, the simple "most frequent class" rule obtains an accuracy similar to NB (0.28), which is not greatly improved by any method. On the other hand, the number of selected predictors is reasonable for SNB, fsNB and CFS + NB, and higher for a $L_{1} / L_{2}-\mathrm{NB}$. The $L_{1} / L_{2}-\mathrm{NB}$ approach (not shown) achieves similar results to $L_{1} / L_{2}-\mathrm{NB}$, for both accuracy and selected variables. The mean number of evaluations for SNB is 30.7, whereas fsNB needs 905.4 evaluations on average.

\subsection{Neuroscience fMRI data}

In this section, we report results on functional magnetic resonance imaging (fMRI) data, the StarPlus data set $^{2}$, collected at Carnegie Mellon University.

Experiments are conducted on six subjects and forty trials per subject. For each trial, the subject is shown a picture for $4 \mathrm{~s}$ and a sentence for $4 \mathrm{~s}$. The objective is to discriminate between these two mental states: "picture" or "sentence". Each data item matches a unique 3-dimensional image. Images are captured every $0.5 \mathrm{~s}$. Hence, each trial has 16 useful images. In brief, there are six data sets, one per subject, and they all have $n=40 \times 16=640$ data items. On the other hand, each image has a number of voxels, split into

\footnotetext{
${ }^{2}$ http://www.cs.cmu.edu/afs/cs.cmu.edu/project/theo-81/www/.
}

25 localized regions of interest (ROIs). In this paper, instead of considering each individual voxel, we will use the mean activation of voxels at each ROI. Therefore, our data set has $p=25$ covariates.

Table 3 shows the results obtained from 10-fold crossvalidation. Algorithms and parameter configuration are the same than in Sects. 7.1 and 7.2.

In this example, fsNB beats the other wrapper algorithms in four out of six subjects, whereas SNB is the best wrapper method for the other two subjects. CFS + NB performs better than fsNB and SNB in one of the subjects.

On the other hand, fsNB selects fewer predictors than SNB in all cases. The number of selected predictors is not very different from CFS + NB.

The performance of $\mathrm{a} L_{1} / L_{2}-\mathrm{NB}$ is poor, and the model selection procedure often prefers the model with no predictors. Note that, in general, none of the approaches behave particularly well. We conjecture that this is because the data have a very nonlinear nature.

The mean number of evaluations for SNB is 126.2, whereas fsNB needs 9,311.1 evaluations on average.

\subsection{Comparison across data sets}

Finally, we perform an overall analysis of the methods that includes the results obtained from all the data sets described above. To do so, we follow the guidelines outlined by [8], performing all pairwise comparisons among the classifiers to detect (statistically) significant differences between each pair. In particular, we use the [2] dynamic procedure to adjust the raw $p$ values. Table 4 shows, for each pair, these adjusted 
Table 4 Adjusted $p$ values, via the Bergmann-Hommel's dynamic procedure, for each pair of methods

\begin{tabular}{llll}
\hline Pair & Adjusted $p$ value & Pair & Adjusted $p$ value \\
\hline fsNB versus NB & $1.57 \mathrm{E}-6$ & SNB versus NB & 0.783 \\
fsNB versus WNB & $3.35 \mathrm{E}-6$ & SNB versus WNB & 0.783 \\
fsNB versus $\mathrm{a} L_{1} / L_{2}$-NB & $3.35 \mathrm{E}-6$ & SNB versus $\mathrm{L} L_{1} / L_{2}$-NB & 0.783 \\
fsNB versus SNB & $9.86 \mathrm{E}-4$ & $\mathrm{CFS}+\mathrm{NB}$ versus SNB & 2.210 \\
fsNB versus CFS + NB & 0.030 & $\mathrm{a} L_{1} / L_{2}$-NB versus NB & 2.424 \\
CFS + NB versus NB & 0.352 & WNB versus NB & 2.424 \\
CFS + NB versus WNB & 0.352 & $\mathrm{a} L_{1} / L_{2}$-NB versus NB & 2.424 \\
CFS + NB versus a $L_{1} / L_{2}$-NB & 0.352 & & \\
\hline
\end{tabular}

$p$ values. We can observe that fsNB is significantly better than all the other procedures, with a significance level of 0.05 .

\section{Discussion}

In this paper, we have proposed a forward stagewise version of the forward stepwise SNB approach. This approach has some advantages over the usual SNB, and often beats other naïve Bayes-based algorithms, like the WNB. We have illustrated this point empirically on both synthetic and real data sets. The forward stagewise approach is computationally more expensive than SNB. Computational complexity, however, can be modulated via the $v$ parameter, which, with $\epsilon$, defines the extent of the search at each step.

We have also extended the $L_{1} / L_{2}$-regularized naïve Bayes approach taken by van Gerven and Heskes [9] to accommodate discrete predictors. In addition, we have introduced a handy modification of this method based on adaptive penalties [19]. Unlike the fsNB, however, the $L_{1} / L_{2}$-regularized naïve Bayes approach does not discard redundant predictors and, hence, performs poorly when the data set contains large sets of these noisy predictors. This phenomenon has been discussed and observed in a comprehensive synthetic experimental setting. $L_{1} / L_{2}$-regularized naïve Bayes fares relatively well, though, when noisy predictors are irrelevant. Nonetheless, irrelevant predictors are considerably less harmful to the classification than redundant predictors.

In addition, note that, whereas it is straightforward for the fsNB approach to deal with data sets with both discrete and continuous predictors, it is not so simple for the $L_{1} / L_{2}$-regularized naïve Bayes method. This is because the continuous and discrete penalties scale differently. Besides discretizing the continuous predictors, we have two choices to address this issue. First, we can use two separate regularization parameters for each type of penalty, which is an expensive solution if they have to be estimated. Second, we can somehow scale the continuous predictors to make the penalties scale similarly. This is an approximate and rather tricky solution, and we do not expect the results to be good.
Also, the WNB approach cannot be used with continuous predictors unless they are discretized beforehand. In summary, flexibility is another advantage of the proposed fsNB approach.

Future work could focus on the possibility of converting the fsNB approach into a boosting method, where all intermediate models collaborate to output a final prediction. Plugging more complex Bayesian classifiers into this framework is also on the agenda. Of course, the algorithm structure accepts other distributions than multinomial and Gaussian.

Acknowledgments Research partially supported by the Spanish Ministry of Science and Innovation, projects TIN2010-20900-C04-04, Consolider Ingenio 2010-CSD2007-00018 and Cajal Blue Brain.

\section{References}

1. Akaike, H.: A new look at the statistical model identification. IEEE Trans. Autom. Control 19, 716-723 (1974)

2. Bergmann, G., Hommel, G.: Improvements of general multiple test procedures for redundant systems of hypotheses. In: Multiple Hypotheses Testing, pp. 100-115. Springer, Berlin (1988)

3. Boyd, S., Vandenberghe, L.: Convex Optimization. Cambridge University Press, Cambridge (2004)

4. Domingos, P., Pazzani, M.: Beyond independence: conditions for the optimality of the simple Bayesian classifier. Mach. Learn. 29, 103-130 (1997)

5. Drugan, M.M., Wiering, M.A.: Feature selection for Bayesian network classifiers using the MDL-FS score. Int. J. Approx. Reason. 51, 695-717 (2010)

6. Ferreira, J.T.A.S., Denison, D.G.T., Hand, D.J.: Data mining with products of trees. In: Advances in Intelligent Data Analysis, Lecture Notes in Computer Science, vol. 2189, pp. 167-176. Springer, Berlin (2001)

7. Friedman, N., Geiger, D., Goldszmidt, M.: Bayesian network classifiers. Mach. Learn. 29, 131-163 (1997)

8. García, S., Herrera, F.: An extension on "statistical comparisons of classifiers over multiple data sets" for all pairwise comparisons. J. Mach. Learn. Res. 9, 2677-2694 (2008)

9. van Gerven, M., Heskes, T.: $L_{1} / L_{p}$ regularization of differences. Tech. Rep. ICIS-R08009, Radboud University Nijmegen (2008)

10. Hall, M.: Induction of selective Bayesian classifiers. In: Proceedings of the 17th International Conference on Machine Learning, pp. 359-366 (2000) 
11. Hand, D.J., Yu, K.: Idiot's Bayes—not so stupid after all. Int. Stat. Rev. 69, 385-398 (2001)

12. Kohavi, R., John, G.H.: Wrappers for feature subset selection. Artif. Intell. 29, 273-324 (1996)

13. Langley, P., Sage, S.: Induction of selective Bayesian classifiers. In: Proceedings of the 10th Conference on Uncertainty in Artificial Intelligence, pp. 399-406 (1994)

14. Minsky, M.: Steps toward artificial intelligence. In: Computers and Thought, pp. 406-450. McGraw-Hill, New York (1961)

15. Tibshirani, R.: Regression shrinkage and selection via the Lasso. J. Royal Stat. Soc. Ser. B 58, 267-288 (1996)
16. Tseng, P.: Convergence of block coordinate descent method for nondifferentiable minimation. J. Optim. Theory Appl. 109, 475494 (2001)

17. Weisberg, S.: Applied Linear Regression. Wiley, New York (1980)

18. Yuan, M., Lin, Y.: Model selection and estimation in regression with grouped variables. J. Royal Stat. Soc. Ser. B 70, 53-71 (2006)

19. Zou, H.: The adaptive Lasso and its oracle properties. J. Am. Stat. Assoc. 101, 1418-1429 (2006) 